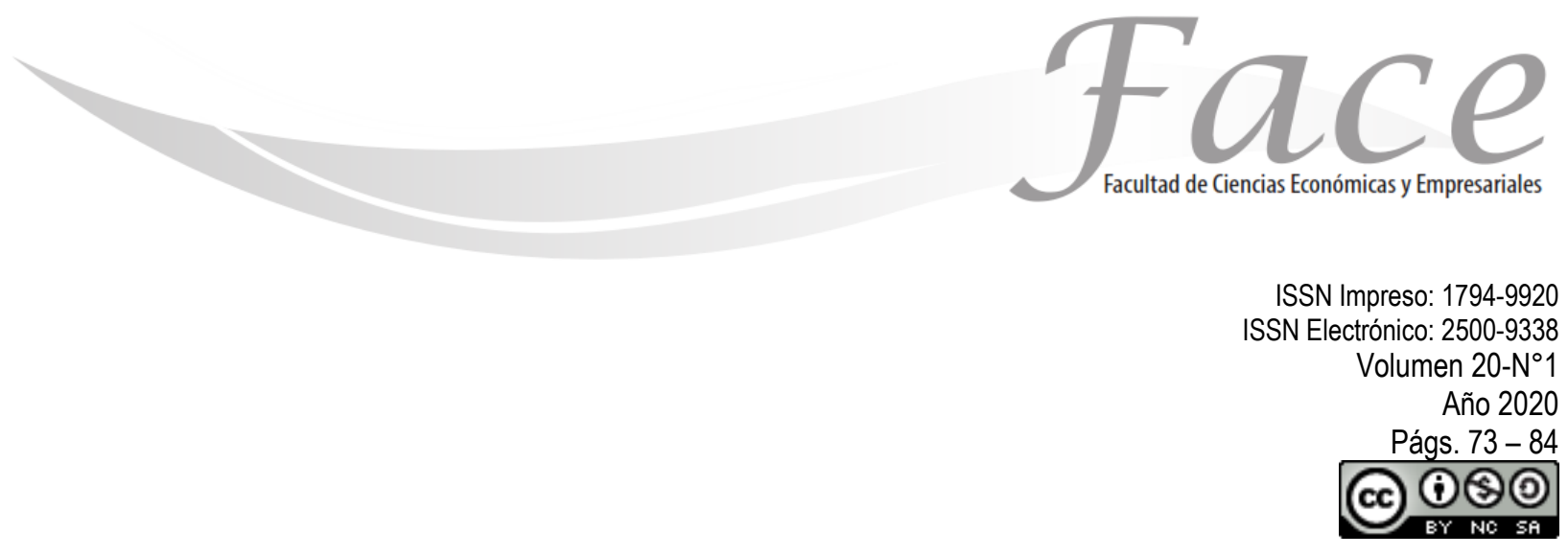

\title{
DOS DÉCADAS DE ESTUDIO DEL CAPITAL INTELECTUAL: UN ESTUDIO BIBLIOMÉTRICO
}

\author{
Alejandra Pulido López* \\ Enlace ORCID: https://orcid.org/0000-0003-2548-3232 \\ Alejandra López Salazar** \\ Enlace ORCID: :https://orcid.org/0000-0003-3927-9603
}

Fecha de Recepción: Agosto 2 de 2020

Fecha de Aprobación: Diciembre 4 de 2020

\section{Resumen:}

El objetivo de este artículo es examinar el estado de la investigación sobre Capítal Intelectual en terminos del comportamiento del tema a los largo de las últimas dos decadas (1997- 2017), con sus variables capital humano, estructural y relacional, para lo cual se realizó un estudio bibliometrico basado en la base de datos de Scopus y teniendo en cuenta el volumen de producción a lo largo del periodo de estudio, el impacto de las publicaciones, journals y autores. El análisis muestra que el estudio en este tema ha incrementado en los últimos veinte años, destacando a Nick Bontis como el autor con mayor volumen de producción e impacto en el estudio del tema y da evidencia de la especialización de dos revistas en Reino Unido, especializados en capital intelectual. Los estudios sobre capital intelectual versan sobre tres áreas principales: la relación del capital intelectual con el desempeño empresarial, medición del capital intelectual y la relación con otras variables como creación de valor, gobierno corporativo, gestión del recurso humano, innovación, entre otros.

Palabras clave: capital intelectual, análisis bibliométrico, desempeño.

\footnotetext{
* Magíster en Comercio Internacional. Profesora de Docencia Escuela de Administración, Colombia. Universidad de Rosario, Colombia . Contacto: alejandra,pulido@uroasario.edu.co

* * Doctora en Negocios y Estudios Económicos. Profesora Investigadora de la División de Ciencias Sociales y Administrativas, México. Universidad de Guanajuato, México .Contacto: alelopez.salazar@yahoo.com
} 


\title{
TWO DECADES OF INTELLECTUAL CAPITAL STUDY: A BIBLIOMETRIC STUDY
}

\begin{abstract}
:
The objective of this article is to examine the state of research on Intellectual Capital in terms of the behavior of the topic over the last two decades (1997-2017), considering its variables human, structural and relational capital, For this purpose, a bibliometric study was carried out based on the Scopus database and taking into account the volume of production over the period of study, the impact of publications, journals and authors. The analysis shows that the study on this topic has increased over the past twenty years, highlighting Nick Bontis as the author with the highest volume of production and impact in the study of the subject and shows evidence of the specialization of two journals in the UK, specializing in intellectual capital. Intellectual capital studies cover three main areas: the relationship of intellectual capital to business performance, measurement of intellectual capital and the relationship with other variables such as value creation, corporate governance, human resource management, innovation, among others.
\end{abstract}

Keywords: intellectual capital, bibliometric analysis, performance.

\section{DUAS DÉCADAS DE ESTUDO DE CAPITAL INTELECTUAL: UM ESTUDO BIBLIOMÉTRICO}

\section{Resumo:}

O objetivo deste artigo é examinar o estado de pesquisa sobre Capítulo Intelectual em termos do comportamento do sujeito nas últimas duas décadas (1997-2017), com suas variáveis capital humano, estrutural e relacional, para o qual foi realizado um estudo bibliométrico com base na base de dados do Scopus e levando em conta o volume de produção ao longo do período de estudo , 0 impacto das publicações, revistas e autores. A análise mostra que o estudo sobre 0 tema aumentou nos últimos vinte anos, destacando Nick Bontis como o autor com maior volume de produção e impacto no estudo do tema e mostra evidências da especialização de duas revistas no Reino Unido, especializadas em capital intelectual. Estudos sobre capital intelectual abrangem três áreas principais: a relação do capital intelectual com 0 desempenho empresarial, a medição do capital intelectual e a relação com outras variáveis como criação de valor, governança corporativa, gestão de recursos humanos, inovação, entre outras.

Palavras-chave: capital intelectual, análise bibliométrica, desempenho 


\section{INTRODUCCIÓN:}

El concepto de capital intelectual fue introducido en 1974 por Locke, quien argumenta que este capital forma parte de la innovación, pero toma fuerza desde la segunda mitad de la década de los 90 s, y aunque en la literatura no existe una definición universal sobre este tema, existen algunos acercamientos y variables que son incluidos por algunos autores quienes han propuesto algunas definiciones, como son los activos intelectuales, factores como el liderazgo en tecnología, el entrenamiento de los empleados, el servicio al cliente, la posición en el mercado, la fidelidad del consumidor (Edvinsson, 1997, p. 3).

El capital intelectual ha sido definido desde dos perspectivas diferentes pero convergentes: la necesidad de aumentar la capacidad competitiva de la empresa y de obtener ventajas estratégicas por el uso intensivo de los recursos intangibles. De estos recursos, se le presta mayor atención a la información y el conocimiento, marcas y patentes, la cultura organizacional y el comportamiento orientado hacia excelencia; esto implica el incremento en la competitividad por un incremento de la eficiencia en la gestión de los recursos (Todericiu y Stăniţ, 2015).

Existen algunos elementos comunes en las definiciones existentes retomados por algunos autores: "la intangibilidad, conocimiento que crea valor y tiene efectos en la práctica colectiva" (Cabrita, 2005, p. 2) y el entendimiento del capital intelectual como el conjunto de valores intangibles que potencian la capacidad de la organización de generar beneficios hoy en día y en el futuro (Soret, 2008).

Alama, Castro y López (2006, p. 3) exponen la definición de Brooking como "combinación de activos inmateriales que permite funcionar a la empresa" $(1997,25)$, y plasman énfasis en la combinación de los intangibles que constituyen el capital intellectual.

Hashim et al. (2012), plantean que en el milenio actual menos personas realizarán trabajo físico y más personas realizarán trabajo de cerebro, denominado capital intelectual, el cual no aparece en el balance de la empresa pero tiene más valor para las organizaciones que los activos físicos. Este concepto es compartido por Sadalia y Lubis (2015), quienes consideran que el capital intelectual es el activo intangible de una organización que es usado para crear valor a la organización mediante la comunicación del capital humano, capital relacional y capital estructural.

Así mismo, estos autores citando a Ulmer (2003) plantean que el capital intelectual no sólo incluye los contenidos de las mentes de los empleados, sino que también incluye la estructura inmaterial y compleja entre ellas y crea las funciones de la organización.

Según Calix, Vigier y Briozzo (2015) los principales elementos distintivos de las organizaciones son los recursos intangibles como el capital intelectual, los cuales crean las condiciones necesarias para llegar a tener ventajas competitivas sostenibles en el tiempo. Bontis por su parte, ha explicado el impacto del capital intelectual en el desempeño de firmas intensivas en servicios y mediciones a nivel empresarial y nacional. (2002, 2007, 2009, 2015).

El estudio de este tema resulta fundamental para comprender las capacidades y oportunidades que presentan las empresas de los sectores industriales y de servicios y servirá para estudios posteriores sobre su medición en contextos empresariales, razón por la cual se genera la necesidad de analizar la evolución del estudio del Capital Intelectual para conocer las tendencias de investigación en el tema y establecer su importancia como campo investigativo en el ámbito empresarial a nivel global.

El objetivo de este artículo es realizar un análisis sobre el estudio de capital intelectual en las dos ultimas décadas, periodo comprendido entre 1997-2017, mediante un análisis bibliométrico apoyado en la base de datos de Scopus.

Así, en el artículo se presentarán los resultados de dicho análisis iniciando con el volumen de la producción y su variación durante el periodo de estudio, seguido del análisis de los países donde más se ha estudiado el tema. Posteriormente se presentará un análisis de las publicaciones con mayor impacto, y las temáticas trabajadas por los autores con mayor volumen de producción e impacto. Finalmente se analizan los journals con mayor producción en el tema de Capital Intelectual. 


\section{METODOLOGÍA}

Se realizó un analisi bibliométrico, teniendo en cuenta la gran importancia que este tipo de estudio ha adquirido en el área de los negocios, en temas como el emprendimiento (Ferrerira, Fernandes y Kraus, 2017; Schmitz, Urbano, Dandolini, de Souza y Guerrero, 2017), la gerencia (Ferrerira, Fernandes y Ratten, 2016; Warner, 2106; Marteens y Guthrie, 2017) y la innovación (Almeida, Santos y Monteiro, 2017; Schmitz, et. al., 2017; Semler, Bortoluzzi y Schenatto, 2015).

Para este análisis se tuvo en cuenta la información obtenida en Scopus, la cual cuenta con 1294 fuentes entre journal, trade journals y series de libro en el área de "Negocios, Gerencia y Contabilidad", area de conocimiento considerada para esta investigación, la cual permite realizar un análisis sobre el volumen de la producción a traves del tiempo, impacto de los autores, journals y artículos en la temática objeto de estudio.

En primer lugar, para la recolección del material, se tuvieron en cuenta las publicaciones realizadas entre 1997 y 2017, considerando los artículos que incluyeran en Título, Abstract y Palabras Clave, los térnimos "capital intelectual", "capital estructural", "capital humano" y "capital relacional", en el área específica de "Negocios, Gerencia y Contabilidad", para excluir publicaciones de áreas de conocimiento no relevantes, con lo cual se creó una lista de 633 publicaciones la cual se clasificó entre artículos de revista, documentos de conferencia, capítulos de libro, reviews, libros, artículo en prensa, conference review y editorial.

Posteriormente se realizó un análisis descriptivo de las publicaciones encontradas respecto al volumen, tendencias en la producción en el periodo de estudio, para posteriormente evaluarlo teniendo en cuenta la procedencia, los autores con mayor producción e impacto y las temáticas trabajadas por dichos autores, y el desarrollo del tema en los artículos de mayor impacto. Finalmente se realizó una descripción y análisis de los journals con mayor impacto.

\section{RESULTADOS:}

Se presentan los resultados generales del análisis bibliométrico teniendo en cuenta el tipo de publicaciones encontradas, el número de publicaciones por año en el periodo analizado, las principales fuentes (journals), los países con mayor producción en el tema, los autores con el mayor número de artículos y citaciones, y las publicaciones con el mayor número de citaciones.

\section{Volumen de la producción}

De las 633 publicaciones encontrados, una gran proporción $(79.9 \%)$ corresponde a artículos con un total de 506 , seguido de 62 documentos de conferencia, 29 capítulos de libro, y 26 revisiones, como se muestra en la siguiente tabla:

Tabla 1. Resultados de la producción

\begin{tabular}{|c|c|c|}
\hline TIPO DE DOCUMENTO & NÚMERO & DE \\
\hline Artículos & & 506 \\
\hline Documentos conferencia & & 62 \\
\hline Capítulo de libro & & 29 \\
\hline Review & & 26 \\
\hline Libro & & 4 \\
\hline Artículo en prensa & & 3 \\
\hline Conference Review & & 2 \\
\hline Editorial & & 1 \\
\hline Total & & 633 \\
\hline
\end{tabular}

Fuente: elaboración propia con base en datos de

$$
\text { Scopus }
$$

\section{Publicaciones a través del tiempo}

Durante las dos décadas estudiadas (1997-2007), se evidencia un incremento en la investigación sobre el capital pasando de dos publicaciones en el año 1997 a 81 en el año 2007. Durante la primera década se encontró un total de 117 publicaciones, mientras que en la segunda se encontraron 515 publicaciones. 
Aunque hay algunos momentos en los que desciende el número de documentos publicados la tendencia muestra un crecimiento positivo en el estudio del tema (figura 1).

Figura1. Publicación por año. índice $\mathrm{H}$ de 21; en segundo lugar se encuentra Canadá con 30 publicaciones, 2,454 citaciones y un índice $\mathrm{H}$ de 17; en tercer lugar Taiwan con 57 publicaciones 2,017 citaciones y presenta el índice $\mathrm{H}$ más alto con 22, seguido de España con 1,274 citaciones y un índice $\mathrm{H}$ de 20 , Malasia con 47 publicaciones 1,153 citaciones y un índice $\mathrm{H}$ de 12 , y finalmente el Reino Unido con 43 documentos, 1,006 citaciones y un índice $\mathrm{H}$ de 17.

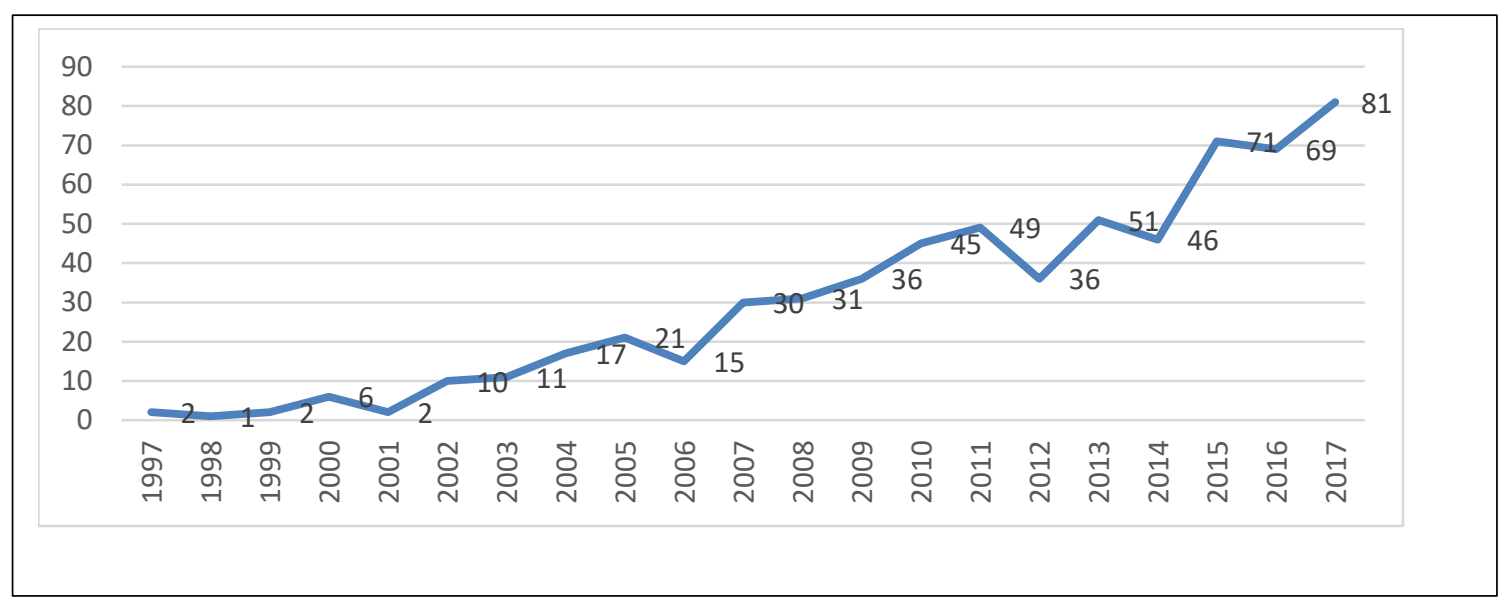

Fuente: elaboración propia con base en datos de Scopus

Tabla 2. Origen de las publicaciones

\section{Países de origen de las publicaciones}

Los diez países con mayor influencia en la producción sobre capital intelectual, generaron 455 publicaciones con por lo menos 100 citaciones. En el top cinco se encuentran España en el primer lugar con 80 publicaciones, seguido de Taiwan con 57, Estados Unidos con 53, Italia 47, Malasia 47 y Reino Unido con 43. Del total de países que han realizado publicaciones sobre capital intelectual, 55 han publicado diez 0 menos artículos en el periodo analizado, en los que se encuentran los países latinoamericanos, siendo Brasil, el país con mayor número de publicaciones (10), seguida de Colombia con cuatro y México con tres (tabla 2).

Respecto al número de citaciones, en este top 10 , a excepción de Irán que muestra 128 citaciones, todos los países cuentan con más de 400 citaciones. Se resaltan los seis países que cuentan con más de mil citaciones. El país con mayor influencia en el tema es Estados Unidos que si bien ocupa el tercer lugar en volumen de producción tiene 3,035 citaciones y un

\begin{tabular}{|c|c|c|c|}
\hline PAÍS & $\begin{array}{c}\text { NUMERO DE } \\
\text { PUBLICACIONES }\end{array}$ & $\begin{array}{l}\text { NUMERO DE } \\
\text { CITACIONES }\end{array}$ & $\begin{array}{c}\text { INDICE } \\
\mathrm{H}\end{array}$ \\
\hline Spain & 80 & 1274 & 20 \\
\hline Taiwan & 57 & 2017 & 22 \\
\hline United States & 53 & 3035 & 21 \\
\hline Italy & 47 & 444 & 12 \\
\hline Malaysia & 47 & 1153 & 12 \\
\hline United Kingdom & 43 & 1006 & 17 \\
\hline Australia & 38 & 690 & 13 \\
\hline Iran & 31 & 128 & 7 \\
\hline Canada & 30 & 2454 & 17 \\
\hline India & 29 & 412 & 8 \\
\hline
\end{tabular}

Fuente: Elaboración propia con base en datos de Scopus 


\section{Publicaciones más citadas}

El total de citaciones de las publicaciones toamdas en cuenta es de 14,240 con un índice H de 57 de los 633 artículos encontrados. Aunque las publicaciones en el tema han incrementado significativamente, el $22.9 \%$ de los artículos nunca han sido citados, el $43 \%$ han sido citados entre una y 10 veces, el $30 \%$ ha sido citado entre 11 y 100 veces, el $3 \%$ ha sido citado entre 100 y 200 veces y el $1 \%$ ha sido citado entre 200 y mil veces (tabla 3). Solamente un artículo ha sido citado más de 1000 veces, el cual corresponde a "The influence of intellectual capital on the types of innovative capabilities (2005)" de los autores Subramaniam y Youndt, con 1073 citas.

Figura 2. Publicaciones más citadas

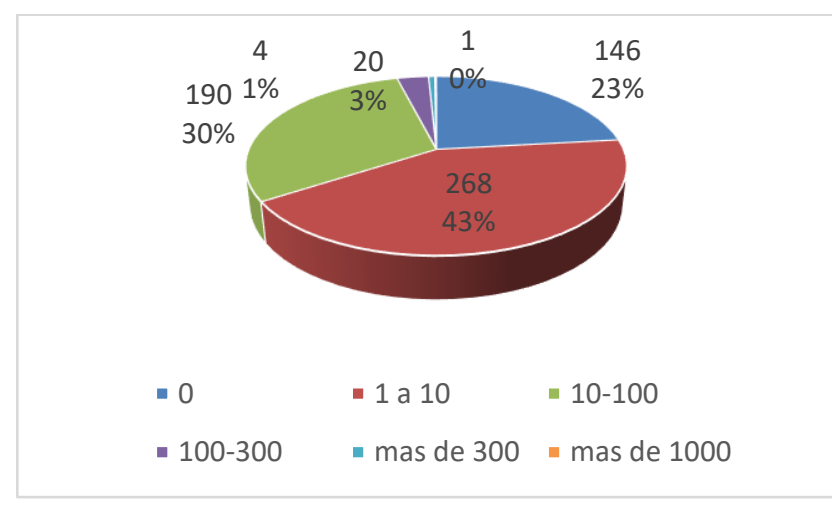

Fuente: elaboración propia con base en datos de

\section{Scopus}

En este artículo se analiza la influencia del capital intelectual en las capacidades de innovación en las organizaciones teniendo en cuenta los componentes del capital intelectual: humano, organizacional y social, acumulando y distribuyendo el conocimiento a través de los individuos, las estructuras, procesos y sistemas organizacionales y las relaciones y redes; y los tipo de capacidades de innovación incremental, entendida como la capacidad para generar innovaciones que refuercen productos y servicios existentes y la innovación radical hacia generación de innovaciones que transformen significativamente productos $y$ servicios existentes.

En el segundo artículo más citado, Intellectual capital and business performance in Malaysian industries, realizado por Bontis, Chua Chong y Richardson, realizan un estudio empírico de los elementos del capital intelectual: humano, estructural y de cliente y su relación con los sectores industriales y de servicios en Malasia. En el tercer artículo Developing intellectual capital at Skandia, Edvinsson realiza una aproximación a la medición del capital intelectual en el modelo desarrollado por Skandia, en el cual se tienen en cuenta los elementos de capital humano y del capital estructural con la implementación de seis fases para su desarrollo.

Con el análisis de los estudios mas citados se determina que un gran grupo de investigaciones se han basado en analizar la influencia del capital intelectual en el desmepeño, rendimiento y en las capacidades de innovación (distintos tipos de innovación), encontrando, en términos generales, que el capital intelectual tiene un impacto positivo en estos elementos (Sharabati, Jawad, Bontis, 2010; Subramaniam y Youndt, 2005; Chen, Cheng y Hwang, 2005; Bontis, Chua Chong y Richardson, 2000; Wang y Chang, 2005). Así mismo, se han analizado estas relaciones de manera específica con cada uno de los componentes del capital intelectual (humano, social y estructural). Estudios como el de Bontis y Fitz-enz (2002) resaltan la importancia del capital humano en el desempeño financiero, mientras que otros estudios muetran que el recurso físico sigue tendiendo mayor influencia en el rendimiento (Firer y Williams, 2003). Es importante resaltar que existen distitntas propuestas en torno a los elementos que componen el capital intelectual, sin que existan grandes diferencias entre ellas, y que los estudios se han llevado a cabo en distintas industrias.

También se tienen estudios que resaltan la importancia del capital intelectual en el proceso de aprendizaje del recurso humano (Keng y Snell, 2009; Hsu y Fang, 2009), así como el imapcto del capital intelectual en el registro contable para mejorar el valor percibido de los stakeholders de la empresa (Abeysekera y Guthrie, 2005), en la capacidad de crear valor en las empresas (Tseng y Goo, 2005), en los factores de gobierno corporativo (Li, Pike y Haniffa, 2008), en la capacidad de desarrollar nuevos productos (Hsu y Fang, 2009), en el comportamiento intraemprendedor (Hayton, 2005), en la sobrevivencia y crecimiento de las empresas (Peña, 2002) y en la facilidad de obrener capital de riesgo (Baum y Silverman, 2004). 
Tabla 3. Publicaciones más citadas

\begin{tabular}{|c|c|c|c|c|}
\hline Title & Author & Year & Source & Cites \\
\hline $\begin{array}{l}\text { The influence of intellectual capital on the types of } \\
\text { innovative capabilities }\end{array}$ & Subramaniam, M., Youndt, M.A. & 2005 & $\begin{array}{l}\text { Academy of Management } \\
\text { Journal }\end{array}$ & 1073 \\
\hline $\begin{array}{l}\text { Intellectual capital and business performance in } \\
\text { Malaysian industries }\end{array}$ & $\begin{array}{l}\text { Bontis, N., William Chua Chong, } \\
\text { K., Richardson, S. }\end{array}$ & 2000 & $\begin{array}{l}\text { Journal of Intellectual } \\
\text { Capital }\end{array}$ & 575 \\
\hline Developing intellectual capital at Skandia & Edvinsson, L. & 1997 & Long Range Planning & 527 \\
\hline $\begin{array}{l}\text { Picking winners or building them? Alliance, intellectual, } \\
\text { and human capital as selection criteria in venture } \\
\text { financing and performance of biotechnology startups }\end{array}$ & Baum, J.A.C., Silverman, B.S. & 2004 & $\begin{array}{l}\text { Journal of Business } \\
\text { Venturing }\end{array}$ & 410 \\
\hline $\begin{array}{l}\text { An empirical investigation of the relationship between } \\
\text { intellectual capital and firms' market value and financial } \\
\text { performance }\end{array}$ & $\begin{array}{l}\text { Chen, M.-C., Cheng, S.-J., Hwang, } \\
\text { Y. }\end{array}$ & 2005 & $\begin{array}{l}\text { Journal of Intellectual } \\
\text { Capital }\end{array}$ & 355 \\
\hline $\begin{array}{l}\text { Intellectual capital ROI: A causal map of human capital } \\
\text { antecedents and consequents }\end{array}$ & Bontis, N., Fitz-enz, J. & 2002 & $\begin{array}{l}\text { Journal of Intellectual } \\
\text { Capital }\end{array}$ & 297 \\
\hline $\begin{array}{l}\text { Intellectual capital and traditional measures of corporate } \\
\text { performance }\end{array}$ & Firer, S., Mitchell Williams, S. & 2003 & $\begin{array}{l}\text { Journal of Intellectual } \\
\text { Capital }\end{array}$ & 266 \\
\hline $\begin{array}{l}\text { National Intellectual Capital Index: A United Nations } \\
\text { initiative for the Arab region }\end{array}$ & Bontis, N. & 2004 & $\begin{array}{l}\text { Journal of Intellectual } \\
\text { Capital }\end{array}$ & 248 \\
\hline $\begin{array}{l}\text { Measuring intellectual capital: A new model and empirical } \\
\text { study }\end{array}$ & Chen, J., Zhu, Z. \& Yuan Xie, H. & 2004 & $\begin{array}{l}\text { Journal of Intellectual } \\
\text { Capital }\end{array}$ & 247 \\
\hline $\begin{array}{l}\text { Intellectual capital architectures and ambidextrous } \\
\text { learning: A framework for human resource management }\end{array}$ & Kang, S.-C., Snell, S.A. & 2009 & $\begin{array}{l}\text { Journal of Management } \\
\text { Studies }\end{array}$ & 185 \\
\hline VAIC - an accounting tool for IC management & Pulic, A. & 2000 & $\begin{array}{l}\text { International Journal of } \\
\text { Technology Management }\end{array}$ & 171 \\
\hline $\begin{array}{l}\text { An empirical investigation of annual reporting trends of } \\
\text { intellectual capital in Sri Lanka }\end{array}$ & Abeysekera, I., Guthrie, J. & 2005 & $\begin{array}{l}\text { Critical Perspectives on } \\
\text { Accounting }\end{array}$ & 166 \\
\hline $\begin{array}{l}\text { Intellectual capital and performance in causal models. } \\
\text { Evidence from the information technology industry in } \\
\text { Taiwan }\end{array}$ & Wang, W.-Y., Chang, C. & 2005 & $\begin{array}{l}\text { Journal of Intellectual } \\
\text { Capital }\end{array}$ & 162 \\
\hline $\begin{array}{l}\text { Intellectual capital and corporate value in an emerging } \\
\text { economy: Empirical study of Taiwanese manufacturers }\end{array}$ & Tseng, C.-Y., Goo, Y.-J.J. & 2005 & R and $D$ Management & 155 \\
\hline $\begin{array}{l}\text { Intellectual capital disclosure and corporate governance } \\
\text { structure in UK firms }\end{array}$ & Li, J., Pike, R., Haniffa, R. & 2008 & $\begin{array}{l}\text { Accounting and Business } \\
\text { Research }\end{array}$ & 147 \\
\hline $\begin{array}{l}\text { Intellectual capital and new product development } \\
\text { performance: The mediating role of organizational } \\
\text { learning capability }\end{array}$ & Hsu, Y.-H., Fang, W. & 2009 & $\begin{array}{l}\text { Technological Forecasting } \\
\text { and Social Change }\end{array}$ & 143 \\
\hline $\begin{array}{l}\text { Intellectual capital and business performance in the } \\
\text { Portuguese banking industry }\end{array}$ & Do Rosário Cabrita, M., Bontis, N. & 2008 & $\begin{array}{l}\text { International Journal of } \\
\text { Technology Management }\end{array}$ & 129 \\
\hline $\begin{array}{l}\text { Intellectual capital and business performance in the } \\
\text { pharmaceutical sector of Jordan }\end{array}$ & $\begin{array}{ll}\text { Sharabati, } & \text { A.-A.A., Jawad, } \\
\text { S.N., Bontis, N. }\end{array}$ & 2010 & Management Decision & 125 \\
\hline $\begin{array}{l}\text { Competing in the new economy: The effect of intellectual } \\
\text { capital on corporate entrepreneurship in high-technology } \\
\text { new ventures }\end{array}$ & Hayton, J.C. & 2005 & $\mathrm{R}$ and $\mathrm{D}$ Management & 121 \\
\hline Intellectual capital and business start-up success & Peña, I. & 2002 & $\begin{array}{l}\text { Journal of Intellectual } \\
\text { Capital }\end{array}$ & 118 \\
\hline
\end{tabular}


Con el análisis de los estudios más citados se determina que un gran grupo de investigaciones se han basado en analizar la influencia del capital intelectual en el desmepeño, rendimiento y en las capacidades de innovación (distintos tipos de innovación), encontrando, en términos generales, que el capital intelectual tiene un impacto positivo en estos elementos (Sharabati, Jawad, Bontis, 2010; Subramaniam y Youndt, 2005; Chen, Cheng y Hwang, 2005; Bontis, Chua Chong y Richardson, 2000; Wang y Chang, 2005). Así mismo, se han analizado estas relaciones de manera específica con cada uno de los componentes del capital intelectual (humano, social y estructural). Estudios como el de Bontis y Fitz-enz (2002) resaltan la importancia del capital humano en el desempeño financiero, mientras que otros estudios muetran que el recurso físico sigue tendiendo mayor influencia en el rendimiento (Firer y Williams, 2003). Es importante resaltar que existen distintas propuestas en torno a los elementos que componen el capital intelectual, sin que existan grandes diferencias entre ellas, y que los estudios se han llevado a cabo en distintas industrias.

También se tienen estudios que resaltan la importancia del capital intelectual en el proceso de aprendizaje del recurso humano (Keng y Snell, 2009; Hsu y Fang, 2009), así como el imapcto del capital intelectual en el registro contable para mejorar el valor percibido de los stakeholders de la empresa (Abeysekera y Guthrie, 2005), en la capacidad de crear valor en las empresas (Tseng y Goo, 2005), en los factores de gobierno corporativo (Li, Pike y Haniffa, 2008), en la capacidad de desarrollar nuevos productos (Hsu y Fang, 2009), en el comportamiento intraemprendedor (Hayton, 2005), en la sobrevivencia y crecimiento de las empresas (Peña, 2002) y en la facilidad de obrener capital de riesgo (Baum y Silverman, 2004).
Otro conjunto de investigaciones se enfocan en realizar propuestas metodológicas para medir el capital inelectual, como el estudio de Edvinsson (1997) y más recientemente el de Chen, Zhu \& Yuan Xie (2004).

\section{Principales autores: top 20}

No se evidencia un alto volumen de publicaciones sobre capital intelectual por autor (tabla 4), a excepción de Bontis quien ha publicado más de diez artículos, mientras que en el top 20, catorce de ellos han publicado entre cinco y diez artículos. El autor con mayor volumen e impacto en el estudio del capital intelectual es Nick Bontis, quien ha publicado 17 artículos en el tema de capital intelectual, 15 en coautoría y dos de manera individual, con 1695 citas y un indice $\mathrm{H}$ de 13 en este tema. Sus artículos presentan principalmente estudios sobre el capital intelectual y el desempeño de negocios en países como Malasia, Portugal, Jordania, Egipto, Pakistán, Serbia y en países árabes y en desarrollo, en sectores como el financiero, hotelero, industrial, en empresas medianas y pequeñas. Bontis ha publicado 114 documentos y cuenta en general con un índice $\mathrm{H}$ de 35.

Miriam Delgado-Verde, por su parte ha escrito ocho artículos en coautoría con 206 citas y un indice $\mathrm{H}$ de 5 , relacionando el capital intelectual y sus componentes con la ventaja competitiva y la innovación en las empresas. Leif Edvinsson, pionero en el estudio del capital intelectual ha escrito siete artículos en este tema desde el punto de vista empresarial y nacional, respecto a su medición e impacto. Las publicaciones en el tema de este autor cuenta con un indice $\mathrm{H}$ de 5 . 
Tabla 4: Principales autores: top 20 e impacto en el estudio del capital intelectual

Fuente: Elaboración propia con base en datos de Scopus

\begin{tabular}{|c|c|c|c|}
\hline Autor & $\begin{array}{c}\text { Documentos } \\
\text { sobre capital } \\
\text { intelectual }\end{array}$ & $\begin{array}{l}\text { Número de } \\
\text { citas }\end{array}$ & Indice $\mathrm{H}$ \\
\hline Bontis, N. & 17 & 1695 & 13 \\
\hline Delgado-Verde, M. & 8 & 219 & 5 \\
\hline Edvinsson, L. & 7 & 741 & 5 \\
\hline Abeysekera, I. & 7 & 319 & 5 \\
\hline Guthrie, J. & 7 & 294 & 5 \\
\hline Khalique, M. & 7 & 38 & 2 \\
\hline Claver-Cortés, E. & 6 & 36 & 3 \\
\hline Lin, C.Y.Y. & 6 & 174 & 4 \\
\hline Akhavan, $\mathrm{P}$. & 5 & 64 & 4 \\
\hline Hamzah, N. & 5 & 3 & 1 \\
\hline Kong, E. & 5 & 11 & 2 \\
\hline Sáenz, J. & 5 & 72 & 3 \\
\hline de Castro, G.M. & 5 & 65 & 3 \\
\hline Jardon, C.M. & 5 & 43 & 1 \\
\hline Sáez, P.L. & 5 & 7 & 1 \\
\hline Aramburu, N. & 4 & 40 & 3 \\
\hline Cleary, $\mathrm{P}$. & 4 & 43 & 4 \\
\hline Cruz-González, J. & 4 & 82 & 3 \\
\hline Dumay, J. & 4 & 35 & 3 \\
\hline
\end{tabular}

\section{Journals más importantes}

Poco más de la tercera parte de los documentos publicados sobre capital intelectual en el periodo de estudio se concentran en cinco journals. En total, 123 journals han publicado los 633 documentos sobre el capital intelectual. Respecto al impacto de estas fuentes, se encontró que los journals de Decisiones Gerenciales y Conocimiento del management son los de mayor impacto. El Journal de Capital intelectual cuenta con el mayor volumen de publicaciones y con mayor impacto en las fuentes de temas muy especializados (46). Y aunque los países de procedencia de los autores es variado, los journals más importantes se encuentran en el Reino Unido.

\section{Tabla 5. Journals más importantes}

\begin{tabular}{|l|l|l|l|l|}
\hline Journal & $\begin{array}{l}\text { Número de } \\
\text { documentos }\end{array}$ & $\begin{array}{l}\text { Índice } \\
\mathbf{H}\end{array}$ & $\begin{array}{l}\text { Citas por } \\
\text { documento }\end{array}$ & $\begin{array}{l}\text { Total } \\
\text { citas }\end{array}$ \\
\hline $\begin{array}{l}\text { Journal Of } \\
\text { Intellectual Capital }\end{array}$ & 139 & 46 & & \\
\hline $\begin{array}{l}\text { International Journal } \\
\text { Of Learning And } \\
\text { Intellectual Capital }\end{array}$ & 51 & 12 & & \\
\hline $\begin{array}{l}\text { Management } \\
\text { Decision }\end{array}$ & 16 & 57 & 1,47 & 600 \\
\hline $\begin{array}{l}\text { Knowledge } \\
\text { Management } \\
\text { Research } \\
\text { Practice And }\end{array}$ & 11 & 24 & 1,34 & 172 \\
\hline $\begin{array}{l}\text { Journal } \\
\text { Knowledge Of } \\
\text { Management }\end{array}$ & 8 & 63 & 2,67 & 648 \\
\hline
\end{tabular}

Fuente: elaboración propia con base en datos de Scopus 


\section{CONCLUSIONES:}

La producción en el tema de capital intelectual ha tenido un incremento significativo en las dos últimas décadas, pasando de dos artículos en 1997 a 81 en el año 2017. Aunque los países más importantes en publicaciones son España, Taiwan, Estados Unidos, Italia y Malasia, las publicaciones más relevantes se han desarrollado en International Journal of Learning and Intellectual Capital y en Journal of Intellectual Capital, los cuales pertenecen al Reino Unido, por lo que se evidencia entonces la concentración de publicaciones en el tema en Europa , Asia y Estados Unidos, encontrando una oportunidad para el estudio del tema en América Latina. Los autores más representativos en este tema son Bontis, Delgado-Verde, Edvinsson, Abeysekera y Guthrie, siendo el primero quien se consolida como el autor más citado con 1695 citas en el periodo de estudio y con mayor impacto en el tema.

También se concluye que el estudio del capital intelectual tiene tres enfoques principales. El primero es la relación del capital intelectual con el desempeño empresarial; el segundo enfoque se concentra en propuestas de medición del capital intelectual en función del contexto en el que se desarrolla la empresa; y, el tercero en establecer relaciones con diversas variables como son el emprendimiento, la creación de valor, la innovación, financiamiento, entre otros.

Este estudio es una base para el análisis del capital intelectual en el contexto empresarial, su medición y relación con la actividad y desempeño de organizaciones de diversos sectores industriales y de servicios.

\section{REFERENCIAS:}

Abeysekera, I. \& Guthrie, J. (2005). An empirical investigation of annual reporting trends of intellectual capital in Sri Lanka. Critical Perspectives on accounting, 16(3), 151-163.
Alama-Salazar, E. M., Martín-de-Castro, G., Navas-López, J. E., \& López-Sáez, P. (2010). Assessing knowledge assets in professional service firms: Proposing a model of intellectual capital. Paper presented at the Knowledge Management and Innovation: A Business Competitive Edge Perspective - Proceedings of the 15th International Business Information Management Association Conference.

Almeida, F.L., Santos, J.D. \& Monteiro, J.A. (2017). A survey of innovation performance models and metrics. Journal of Applied Economic Sciences, XII (6), 1732-1750.

Baum, J.A.C. \& Silverman, B.S. (2004). Picking winners or building them? Alliance, intellectual, and human capital as selection criteria in venture financing and performance of biotechnology startups. Journal of Business Venturing, 19(3), 411-436.

Bontis, N. (2004). National Intellectual Capital Index: A United Nations initiative for the Arab region. Journal of Intellectual Capital, 5 (1), 13-39.

Bontis, N. \& Fitz-enz, J. (2002). Intellectual capital ROI: A causal map of human capital antecedents and consequents. Journal of Intellectual Capital, 3(3), 223-247.

Bontis, N., Janošević, S. \& Dženopoljac, V. (2015). Intellectual capital in Serbia's hotel industry. International Journal of Contemporary Hospitality Management, 27 (6), 1365-1384. DOI: 10.1108/IJCHM-12-2013-0541

Bontis, N., Seleim, A. \& Ashour, A. (2007). Human capital and organizational performance: A study of Egyptian software companies. Management Decision, 45(4), 789-801. DOI: $10.1108 / 00251740710746033$

Bontis, N. \& Serenko, A. (2009). A causal model of human capital antecedents and consequents in the financial services industry. Journal of Intellectual Capital, 10(1), 53-69. DOI: $\underline{10.1108 / 14691930910922897}$

Bontis, N., Chua Chong, W. \& Richardson, S. (2000). Intellectual capital and business performance in Malaysian industries. Journal of Intellectual Capital, 14(1), 29-45. 
Calix, C., Vigier H. \& Briozzo, E. (2015) Capital intelectual y otros determinantes de la ventaja competitiva en empresas exportadoras de la zona norte de Honduras, Suma de Negocios, 6 (14), 130-137. http://dx.doi.org/10.1016/j.sumneg.2015.10.005

Chen, M., Cheng, S. \& Hwang, Y. (2005). An empirical investigation of the relationship between intellectual capital and firms' market value and financial performance. Journal of Intellectual Capital, 6(2), 159176. https://doi.org/10.1108/146919305105927 71

Chen, J., Zhu, Z. \& Yuan Xie, H. (2004). Measuring intellectual capital: A new model and empirical study. Journal of Intellectual Capital, 5(1), 195212. https://doi.org/10.1108/14691930410513003

Delgado-Verde, M., Cooper, S. \& Castro, G. (2015). The moderating role of social networks within the radical innovation process: A multidimensionality of human capital-based analysis. International Journal of Technology Management, 69(2), 117138.

Delgado-Verde, M., Martín De Castro, G., Navas-López, J. \& Amores-Salvadó, J. (2014). Vertical relationships, complementarity and product innovation: An intellectual capital-based view. Knowledge Management Research and Practice, 12(2),226-235. https://doi.org/10.1057/kmrp.2012.59

Delgado-Verde, M., Martín-De Castro, G. \& Amores-Salvadó, J. (2016). Intellectual capital and radical innovation: Exploring the quadratic effects in technology-based manufacturing firms. Technovation, 54, 35-47. http://dx.doi.org/10.1016/j.technovation.2016.02 .002

Delgado-Verde,M., Martín-de-Castro, G., Navas-López, J. \& Cruz-González, J. (2011). Capital social, capital relacional e innovación tecnológica. una aplicación al sector manufacturero español de alta y media-alta tecnología. Cuadernos de Economia y Direccion de la Empresa, 14(4), 207-221.

Delgado-Verde, M., Navas-López, J.E., Cruz-González, J. \& Amores-Salvadó, J. (2011). Radical innovation from relations-based knowledge: Empirical evidence in Spanish technology-intensive firms. Journal of Knowledge Management, 15, 722737.
Do Rosário, M. \& Bontis, N. (2008). Intellectual capital and business performance in the Portuguese banking industry. International Journal of Technology Management, 43, 1-3, 212-237.

Edvinsson, L. (2000). Some perspectives on intangibles and intellectual capital 2000. Journal of Intellectual Capital, 1(1), 12-16. DOI: $\underline{10.1108 / 14691930010371618}$

Edvinsson, L. (1997). Developing intellectual capital at Skandia. Long Range Planning, 30(3), 366-373. https://doi.org/10.1016/S0024-6301(97)90248-X

Ferreira, J., Fernandes, C. \& Kraus, S. (2017). Entrepreneurship research: mapping intellectual structures and research trends. Review of Managerial Science, 13(2), 181-205. DOI: 10.1007/s11846-017-0242-3

Ferreira, J., Fernandes, C. \& Ratten, V. (2016). A co-citation bibliometric analysis of strategic management research. Scientometrics, 109(1), 1-32.

Firer, S. \& Mitchell Williams, S. (2003). Intellectual capital and traditional measures of corporate performance. Journal of Intellectual Capital. 4(3), 348-360. https://doi.org/10.1108/14691930310487806

Hashim, F., Jaffar, R., Hassan, M. S., \& Shukor, Z. A. (2012). The role of diversification on the relationship between intelectual capital disclosure and firm value. Journal Pengurusan, 35, 43-55.

Hayton, J. (2005). Competing in the new economy: The effect of intellectual capital on corporate entrepreneurship in high-technology new ventures. R\&D Management, 35(2), 137-155.

Hsu, Y. \& Fang, W. (2009). Intellectual capital and new product development performance: The mediating role of organizational learning capability. Technological Forecasting and Social Change, $\quad$ 76(5), 664-677. https://doi.org/10.1016/j.techfore.2008.03.012

Kang, S. \& Snell, S. (2009). Intellectual capital architectures and ambidextrous learning: A framework for human resource management. Journal of Management Studies, 46(1), 65-92. DOI: $10.1111 /$ j.1467-6486.2008.00776.x

Li, J., Pike, R. \& Haniffa, R. (2008). Intellectual capital disclosure and corporate governance structure in UK firms. Accounting and Business Research, 38(2),

137-159. 
ISSN: 1794-9920 Impreso / Electrónico 2500-9338

Agosto - Diciembre. Volumen 20 Número 2, Año 2020 Págs. 73-84

https://doi.org/10.1080/00014788.2008.966332 $\underline{6}$

Lock Lee, L. \& Guthrie, J. (2010). Visualising and measuring intellectual capital in capital markets: A research method. Journal of Intellectual Capital, 11(1), 422. DOI: $\underline{10.1108 / 14691931011013307}$

Martens, M. \& Carvalho, M. (2017). Key factors of sustainability in project management context: $A$ survey exploring the project managers' perspective. International Journal of Project Management. 35(6), 1084-1102. https://doi.org/10.1016/j.ijproman.2016.04.004

Peña, I. (2002). Intellectual capital and business start-up success. Journal of Intellectual Capital, 3(2), 180-198. DOI: $10.1108 / 14691930210424761$

Pulic, A. (2000). VAIC an accounting tool for IC management. International Journal of Technology Management, 20(5), DOI: $10.1504 /$ IJTM.2000.002891

Sadalia,I. \& Lubis,A. (2015). Discriminant analysis of intellectual capital model of State University in Medan. Docial and Behavioral Sciences, 211, 476-480.

https://doi.org/10.1016/j.sbspro.2015.11.063

Schmitz, A., Urbano, D., Dandolini, G.A., de Souza, J.A. \& Guerrero, M. ( 2017). Innovation and entrepreneurship in the academic setting: a systematic literature review International Entrepreneurship and Management Journal. 13(2), 369-395. DOl: 10.1007/s11365016-0401-z

Semler, R., Bortoluzzi, S \& Schenatto, F. (2015). Innovation performance evaluation on company networks: Bibliometric and systemic analysis of international scientific literature | [Avaliação de desempenho da inovação em redes de empresas: Análise bibliométrica e sistêmica da literatura científica internacional. Espacios. 36(24), 3.

Sharabati, A., Jawad, S. \& Bontis, N. (2010). Intellectual capital and business performance in the pharmaceutical sector of Jordan. Management Decision, 48(1), 105-131. DOI $10.1108 / 00251741011014481$

Soret, I. (2008). Modelo de medición de conocimiento y generación de ventajas competitivas sostenibles en el ámbito de la iniciativa ECR. Disertación doctoral. Publicada en Business \& Economics Jul 26, 2008. ESIC.

Subramaniam, M. \& Youndt, M. (2005). The influence of intellectual capital on the types of innovative capabilities. Academy of Management Journal, 48(3), 450-463, DOI: $10.5465 / A M J .2005 .17407911$

Todericiu, R. \& Stăniţ, A. (2015). Intelectual Capital - The Key for Sustainable Competitive Advantage for the SME's Sector, Procedia Economics and Finance, 27, 676-681. http://dx.doi.org/10.1016/S22125671(15)01048-5.

Tseng, C. \& Goo, Y.(2005). Intellectual capital and corporate value in an emerging economy: Empirical study of Taiwanese manufacturers. R\&D Management, $\quad 35(2), \quad$ 187-201. https://doi.org/10.1111/j.14679310.2005.00382.x

Wang, W. \& Chang, C. (2005). Intellectual capital and performance in causal models. Evidence from the information technology industry in Taiwan. Journal of Intellectual Capital, 6(2), 222-236. DOI: $10.1108 / 14691930510592816$

Warner, M. (2016). A life cycle of management ideas: A research note. Journal of General Management, 42(2), 17-29.

Yeh-Yun Lin, C. \& Edvinsson, L. (2008). National intellectual capital: Comparison of the Nordic countries. Journal of Intellectual Capital, 9(4), 525-545. DOI: $\underline{10.1108 / 14691930810913140}$ 